\title{
ELUCIDATION OF ELECTROMAGNETIC WAVE EFFECT AND OUTGOING OF FUTURE TREND IN MICROWAVE CHEMISTRY AND BIOLOGY
}

\section{S. Horikoshi}

Sophia University, 7-1 Kioi-cho, Chiyoda-ku, Tokyo

horikosi@sophia.ac.jp

Keywords: Electromagnetic field, semiconductor microwave generator, organic synthesis, photocatalyst, plant breeding, intelligent cooker, aged beef

\begin{abstract}
Can we use microwaves as electromagnetic energy in the chemical and biological fields? We have been researching this question in many aspects and in a bird's-eye view. In response to this question, in some chemical reactions, it turned out that the advantage of microwaves is heat. Note that we also found that this efficient thermal energy transfer method is useful in the chemical field. On the other hand, it was found that electromagnetic waves other than thermal energy can be used in limited chemical reactions (mainly radical reactions). On the other hand, in biological use, there are not many things that require more heat energy than the atmosphere in the first place. However, we have found that depending on the microwave irradiation conditions, it can be activated as a stimulus other than heat. In other words, we discovered a phenomenon that would not have appeared without microwaves. In this paper, it will explain the electromagnetic wave effects of microwaves using photocatalysts, enzymes, and plants as examples. Furthermore, the importance of microwave control will be explained from the development of intelligent cooker.
\end{abstract}

\section{Introduction}

The German chemist Theodor Grotthuss was the first to formulate the first law of photochemistry in 1817; he postulated that a reaction could be driven by light when the energy of light is absorbed by molecules [1]. Grotthuss' idea remained unknown for many years until John W. Draper, an expert in chemistry and photographic processes, proposed in 1842 that only absorbed light rays can produce chemical changes. For this reason, Grotthuss' postulate is better known today as the Grotthuss-Draper law or the first law of photochemistry [2]. The photographic technique developed by Louis Jacques Mandé Daguerre in 1839 as a practical photographic method was to become the starting point of photochemical developments. In this regard, the first researcher to develop true photochemical concepts that distinguished between primary and secondary processes taking place in a chemical system under light absorption was Johannes Stark (1908), who defined the primary process as the immediate absorption of a photon by a molecule or an atom followed by secondary processes. In effect, the law states that in a photochemical process (such as a photochemical reaction) one photon that is absorbed by a molecule causes the main photochemical process. In some circumstances, a molecule having absorbed a photon initiates a process that may involve several other molecules. The Stark-Einstein law is the second law of photochemistry named after Johannes Stark and Albert Einstein. This law states that for each photon of light absorbed by a chemical system, no more than one molecule is activated for a photochemical reaction, as defined by the quantum yield [2]. These two laws have elevated photochemistry as an academic (science) discipline over the last one hundred years. In addition, because of advances in light sources 
and various devices (engineering), such materials and processes as photocatalysts, organic solar cells, photopolymerization, quantum dots, and photochromism (among others) are currently being applied in various other fields.

The next significant surge in chemistry is microwave chemistry wherein microwaves, which represent electromagnetic waves other than light, were introduced as a driving force in the chemical reaction domain in the late 1980s. Since then, thousands of articles have appeared that pertain to microwave-assisted organic syntheses. There are three characteristics in this chemistry when using microwaves. The first is the high heating efficiency caused by the energy of the microwaves that directly reach and are absorbed by the substance. The second is the selectivity with which a specific substrate is heated, while the third characteristic is the enhancement of chemical syntheses by the microwaves' electromagnetic wave energy, often referred to as the microwave effect (or non-thermal effect). Together with the first and second efficient heat effects, these open a new path in microwave chemistry (see e.g., ref. 3 ). The phenomenon of the microwave effect (third characteristic) impacting chemical reactions has been summarized in much of the relevant literature (see e.g., ref. 4). Many researchers who have tackled the mechanism suggest either the presence or the absence of the microwave effect [5]. The reason why the microwave effect has not been clarified to anyone's satisfaction is that the term microwave effect in microwave chemistry includes numerous factors. Accordingly, the microwave phenomenon, which is not yet well understood, is bundled by the word microwave effect and thus has failed to being classified properly.

Thermal energy can be exchanged instantaneously via several mechanisms such as dipolar polarization, ionic conduction, and the Maxwell-Wagner effect (among others) when a substance absorbs microwaves. Consequently, is the microwave involvement in chemical reactions an electromagnetic wave effect (non-thermal effect) or is it a temperature effect (thermal effect)? Experience has shown that it is difficult to separate between the two. Such problems do not occur in photochemistry. Moreover, microwaves (e.g., at a frequency of $2.45 \mathrm{GHz}$; wavelength $=12.24 \mathrm{~cm}$ ) cause electromagnetic waves to be distributed unevenly in the sample, thereby resulting in a non-uniform heating of the sample [6]. Hence, it is necessary to have a proper appreciation of microwave engineering (e.g., fabrication of a microwave apparatus) and to pay close attention to sample size and microwave irradiation methods. Failure to do so can result in erroneous reporting of microwave effects originating from incorrect temperature measurements. Consequently, it is necessary to implement the following points in order to clarify the microwave effect: (i) elucidate the meaning of the microwave effect, (ii) establish an experimental system from which we can observe the microwave effect on the sample using electromagnetic waves and heat, and (iii) build an experimental system (microwave engineering) such that measurement errors of temperatures are avoided.

To achieve some of the above points, we have examined photocatalyzed reactions enhanced by microwaves for nearly two decades during which we demonstrated the microwave effect by simultaneously irradiating the metal-oxide photocatalyst $\mathrm{TiO}_{2}$ with UV light and microwave radiation in reactions taking place during wastewater treatments, something that could not and cannot be achieved by conventional heating [7]. As a non-thermal effect, the microwave effect in photocatalyzed reactions was demonstrated by establishing that the lifetime and utilization efficiency of electrons excited by UV light in the photocatalyst are enhanced by the microwave radiation [8]. Other researchers have also investigated such phenomena. For instance, Kishimoto and coworkers reported that an applied microwave 
field can enhance the photocatalytic reduction of bipyridinium ion using $\mathrm{CdS}$ quantum dots (QDs) via an acceleration of the electron transfer process [9].

In this paper, the use of "microwave energy is introduced as electromagnetic energy" in the chemical, environmental, biological and food fields, taking photocatalytic reactions as an example. On the other hand, microwaves can deliver energy to matter in pulses [10]. The pulsed microwave irradiation will also deal with various phenomena caused by irradiation in chemical reaction and enzyme reaction. The use of microwave energy as these electromagnetic waves is made possible by using a semiconductor generator.

\section{Results and discussions}

\subsection{Coupled Microwave/Photoassisted Methods for Environmental Remediation}

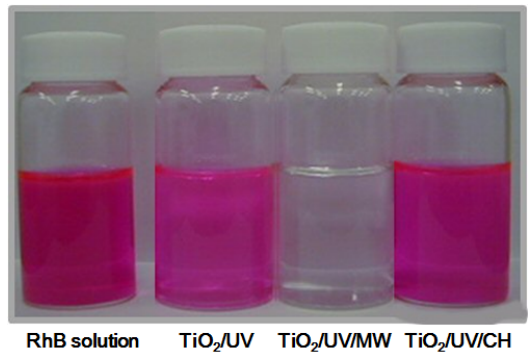

Figure 1. Visual comparison of color fading in the degradation of RhB solutions $(0.05 \mathrm{mM})$ subsequent to being subjected to various degradation methods for $150 \mathrm{~min}$.

From left to right: initial RhB solution; RhB subjected to photoassisted degradation (UV); RhB subjected to integrated microwave-/photo-assisted degradation (UV/MW); $\mathrm{RhB}$ subjected to thermal- and photo-assisted degradation $(\mathrm{UV} / \mathrm{CH})$. Reproduced from [18]. Copyright 2009 by Elsevier B.V.

The photoassisted oxidative (and reductive) decomposition of pollutants by means of $\mathrm{TiO}_{2}$ semiconductor nanoparticulates is an effective and attractive oxidation (reduction) method in the general area of Advanced Oxidation Technologies. Several review articles have appeared that summarize environmental protection using $\mathrm{TiO}_{2}$ materials as the photomediators, if not as photocatalysts [11-14]. Applications of photoassisted treatments to air pollution have been developed by $\mathrm{TiO}_{2}$ fixation on such suitable substrate supports as filters in air conditioners, for instance [15]. However, this photoassisted degradation methodology is not suitable for large-scale wastewater treatment because the degradation rates of organic compounds dissolved in wastewaters tend to be rather slow. In this regard, relatively little has been done in this area in the last several decades as large-scale treatments of organic pollutants in aquatic environments have not been without some problems, not least of which is the low photodegradation efficiency, a result of several factors.

The rate of photodegradation of the cationic dye rhodamine- $\mathrm{B}(\mathrm{RhB})$ is slow in acidic aqueous media because the surface of $\mathrm{TiO}_{2}$ particles is positively charged $\left(\mathrm{Ti}-\mathrm{OH}_{2}{ }^{+}\right.$; $\mathrm{pI}=6.3$ ). However, $\mathrm{RhB}$ has proven as an interesting model compound to examine the microwave effect. In earlier studies, our major focus was on the degradation of organic pollutants, as exemplified by the degradation of the rhodamine- $\mathrm{B}(\mathrm{RhB})$ dye catalyzed by $\mathrm{TiO}_{2}$ semiconductor particles under both UV and microwave irradiation $[16,17]$. Changes in color intensity of the $\mathrm{RhB}$ dye solutions occurring under various conditions are illustrated in Figure 1. The photodegradation of $\mathrm{RhB}$ is clearly evident on using the $\mathrm{TiO}_{2}$-assisted $\mathrm{UV}$ 
and microwave (UV/MW) method. These observations demonstrate that a method that can treat large quantities of pollutants in wastewaters by a hybrid combination of microwaves and $\mathrm{TiO}_{2}$ photoassisted technologies is conceivable. The photodegradation by this metal oxide is unaffected by conventional heating $(\mathrm{CH})$; compare, for example, the results from the UV and the $\mathrm{UV} / \mathrm{CH}$ methods in the presence of $\mathrm{TiO}_{2}$ (Figure 1) [18].

An integrated microwave-/photoassisted methodology presents certain advantages in wastewater treatment. In this technique, a feature of the reaction on the $\mathrm{TiO}_{2}$ surface involves thermal and specific effects (e.g., non-thermal effects) originating from the absorption of microwave radiation by the metal-oxide nanoparticulates. Differences between various $\mathrm{TiO}_{2}$ batches with regard to microwave-specific effect(s) were examined using microwaves of different frequencies [19], and by examining the effects of the microwaves' magnetic and electric fields [20] in photoassisted processes involving $\mathrm{TiO}_{2}$ and $\mathrm{ZnO}$ nanomaterials. The microwave specific effect, not encountered when the photocatalyzed reaction is subjected to conventional heating, inferred that the composition and the electronic characteristics of the $\mathrm{TiO}_{2}$ nanomaterials were important factors. We researched from various angles other than this. Among them, what is particularly remarkable is that they are not responsive to microwaves except for Evonik $\mathrm{P} 25 \mathrm{TiO}_{2}$ particle (commerciale $\mathrm{TiO}_{2}$ particles).

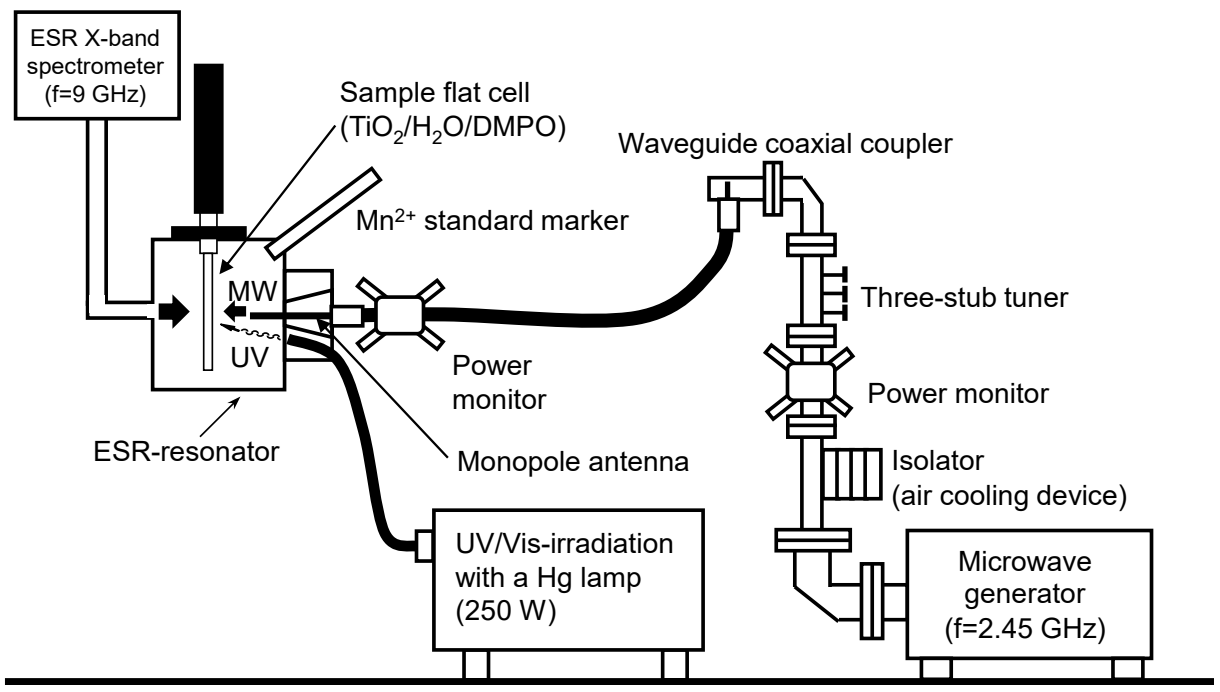

Figure 2. Setup used to generate $\cdot \mathrm{OH}$ radicals in water alone under $\mathrm{MW}$ irradiation, in an aqueous $\mathrm{TiO}_{2}$ dispersion by $\mathrm{MW}$ irradiation alone, and by the UV and UV/MW methods. Reproduced from [21]. Copyright 2003 by Elsevier B.V.

For the photocatalytic reaction mechanism, Titanium dioxide has a bandgap of $3.20 \mathrm{eV}$ (anatase crystal), which corresponds to a wavelength of $387 \mathrm{~nm}$. This means that electronhole pairs are created when $\mathrm{TiO}_{2}$ is radiated with UV-light with wavelengths shorter than $387 \mathrm{~nm}$ (Reaction (1)). To the extent that not all the photogenerated electrons and holes recombine, some of the holes can migrate to the surface and react with surface-bound -OH groups and/or water molecules surrounding the particles that lead ultimately to the formation of hydroxyl radicals (Reaction (2)). Dissolved oxygen molecules react with conduction band electrons $\left(\mathrm{e}^{-}\right)$to yield superoxide radical anions $\left(\mathrm{O}_{2}^{-\bullet}\right.$; 
Reaction (3)), which on protonation generate the hydroperoxy radicals ${ }^{\bullet} \mathrm{OOH}$ (Reaction (4)). Accordingly, the photooxidation of organic substrates with the $\mathrm{UV} / \mathrm{TiO}_{2}$-driven photoassisted process (Reaction (5)) depends on the concentration of ${ }^{\bullet} \mathrm{OH}\left(\right.$ and/or $\left.{ }^{\bullet} \mathrm{OOH}\right)$ radicals produced by the photooxidation of surface hydroxyl groups and/or chemisorbed $\mathrm{H}_{2} \mathrm{O}$. Here, the following questions arise. Does the amount of ${ }^{\bullet} \mathrm{OH}$ radicals generated from $\mathrm{TiO}_{2}$ change under microwave irradiation?

$$
\begin{aligned}
& \mathrm{TiO}_{2}+\mathrm{h} v \rightarrow \mathrm{TiO}_{2}\left(\mathrm{e}^{-}+\mathrm{h}^{+}\right) \rightarrow \mathrm{e}^{-}+\mathrm{h}^{+} \\
& \mathrm{h}^{+}+{ }^{-} \mathrm{OH}_{\text {surf. }}\left(\text { and } / \text { or } \mathrm{H}_{2} \mathrm{O}\right) \rightarrow{ }^{\bullet} \mathrm{OH}\left(+\mathrm{H}^{+}\right) \\
& \mathrm{e}^{-} \text {cb }+\mathrm{O}_{2} \rightarrow \mathrm{O}_{2}^{-} \cdot \\
& \mathrm{O}_{2}{ }^{-}+\mathrm{H}^{+} \rightarrow \cdot{ }^{\bullet} \mathrm{OOH}
\end{aligned}
$$

$\bullet \mathrm{OH}\left(\right.$ or $\left.^{\bullet} \mathrm{OOH}\right)+$ organic pollutant $\rightarrow$ Oxidative products

The possible enhancement of the photoactivity of metal-oxide specimens subsequent to being exposed to microwave radiation from the viewpoint of the amount of - $\mathrm{OH}$ radicals generated was also investigated [21]. Formation of ${ }^{\bullet} \mathrm{OH}$ radicals during $\mathrm{TiO}_{2}$ assisted photooxidations that were driven simultaneously by UV light and microwave radiation was probed by electron spin resonance spectroscopy employing a novel setup in which the ESR sample (contained the DMPO spin-trap agent and $\mathrm{TiO}_{2}$ particles in aqueous media) was irradiated by both UV light and microwave radiation [21]. Figure 2 shows a special ESR system that can observe ${ }^{\bullet} \mathrm{OH}$ radicals in situ while simultaneously irradiating microwaves and ultraviolet light.

The number of ${ }^{\bullet} \mathrm{OH}$ radicals generated under various experimental conditions is summarized in Table 1. For P25 titania, the number of ${ }^{\circ} \mathrm{OH}$ radicals produced by the UV/MW method was nearly $30 \%$ greater than the quantity generated by the UV method alone [21]. A fivefold increase in incident microwave power from 3 to $16 \mathrm{~W}$ led to a significant increase ( $\mathrm{ca} .40 \%)$ in the number of ${ }^{\circ} \mathrm{OH}$ radicals. Such an increase was sufficient to increase the efficiency of the photooxidation of the organic pollutant in water.

Table 1. Number of DMPO- ${ }^{\circ} \mathrm{OH}$ spin adducts produced in the various heterogeneous systems under microwave irradiation, UV irradiation, and $\mathrm{MW} / \mathrm{UV}$ irradiation relative to those formed in the rutile $\mathrm{TiO}_{2}$ specimen for the $\mathrm{TiO}_{2} / \mathrm{H}_{2} \mathrm{O} / \mathrm{MW}$ heterogeneous system. Reproduced from [21]. Copyright 2003 by Elsevier B.V.

\begin{tabular}{|c|c|c|c|c|}
\hline & P25 & UV100 & Anatase & Rutile \\
\hline UV & 182 & 45 & 110 & 110 \\
\hline $\begin{array}{c}\text { UV/MW } \\
(3 \mathrm{~W})\end{array}$ & 259 & 51 & 92 & 76 \\
\hline $\begin{array}{c}\text { UV/MW } \\
(16 \mathrm{~W})\end{array}$ & 369 & - & - & - \\
\hline
\end{tabular}


For the UV100 $\mathrm{TiO}_{2}$ sample (anther commercial $\mathrm{TiO}_{2}$ particles), the increase in the number of ${ }^{\bullet} \mathrm{OH}$ radicals produced was only $10 \%$ greater on increasing the MW power five times. On the other hand, the number of ${ }^{\bullet} \mathrm{OH}$ radicals generated for the pristine anatase and rutile $\mathrm{TiO}_{2}$ samples decreased under microwave irradiation. The P25 specimen was clearly influenced by the microwaves and generated ${ }^{\bullet} \mathrm{OH}$ radicals efficiently under the influence of microwave effects. Therefore, the rate of decomposition was enhanced when P25 was used to decompose the wastewater sample by the UV/MW method. On the other hand, to the extent that the quantity of $\bullet \mathrm{OH}$ radicals produced by the other $\mathrm{TiO}_{2}$ does not increase even when irradiated with microwaves, the rate of decomposition is not enhanced.

Why can only Evonik P25 $\mathrm{TiO}_{2}$ particles benefit from microwaves? P25 might be a hint that it is a mixed crystal of anatase and rutile. The effects of $2.45-\mathrm{GHz}$ microwave radiation on the Raman-active lattice phonons of two selected (Wako) anatase and rutile specimens and on Degussa P-25 $\mathrm{TiO}_{2}$ nanoparticles were probed by in-situ microscopic Raman spectroscopy in the solid phase with samples subjected to microwave irradiation (MW) or in combination with UV illumination (UV/MW) [22]. Significant changes were seen in the Raman band intensities for the pure anatase $E_{g}$ mode at $143 \mathrm{~cm}^{-1}$ and for the rutile vibration at $446 \mathrm{~cm}^{-1}$, whereas only negligible changes in intensity were observed for the $144 \mathrm{~cm}^{-1}$ band of P-25 nanoparticles exposed to MW and UV/MW radiation. It is deduced that microwaves have a negligible impact on the lattice vibrational modes (phonons) of P-25 titania, even though this specimen was thermally heated by the microwaves. The photodegradation of phenol was re-visited to examine the photoactivity of the $\mathrm{TiO}_{2}$ specimens selected for the $i n$-situ Raman study, as attested by the number of $\bullet \mathrm{OH}$ radicals produced (DMPO spin trap ESR) under the UV versus UV/MW irradiations, and by the related enhanced dynamics under UV/MW irradiation relative to $\mathrm{UV}$ alone or $\mathrm{UV} / \mathrm{CH}$. Figure 3 shows a special Raman spectroscopy system that can observe Raman spectral radicals in situ while simultaneously irradiating microwaves and ultraviolet light.

Scheme 1 illustrates a simplistic view of the proposed effects of microwave and microwave/ultraviolet irradiations on the anatase and rutile polymorphs. Under MW/dark conditions, we deduce that the Raman intensities decrease owing to formation of oxygen vacancies $\left(V_{O}\right)$ induced by the microwave radiation fields as attested for the $\mathrm{P}-25 \mathrm{TiO}_{2}$ sample yielding $F$-type color centers [23]. By contrast, under MW/UV irradiation the Raman intensities promptly increase at first, owing to the UV photogenerated mobile charge carriers in the conduction band (electrons) that lead to an overall increase in polarizability, subsequent to which the intensity ratios decrease back to ca. 1 for anatase and close to 1 for rutile owing to opposing effects between generation of photoelectrons by UV and formation of oxygen vacancies (or other defects) by the microwave radiation fields that trap the electrons to form color centers such as $F$-centers and $\mathrm{Ti}^{3+}$ centers [23]. 


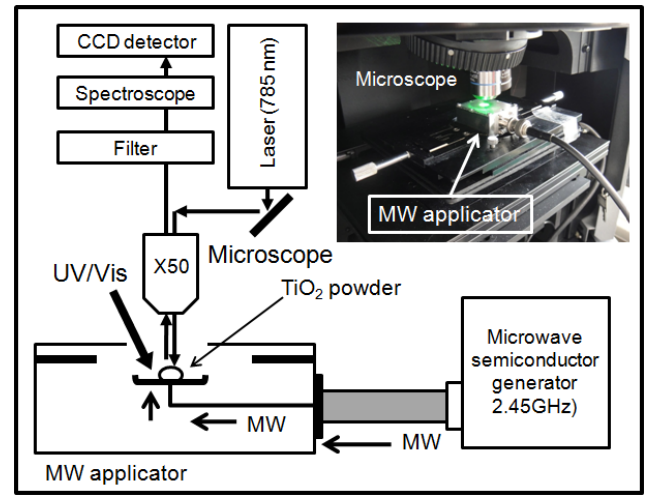

Figure 3. Experimental setup for the in-situ Raman spectroscopy of $\mathrm{TiO}_{2}$ powdered specimens using an integrated Microwave and UV-Vis radiation system. Reproduced from [22]. Copyright 2003 by Elsevier B.V.

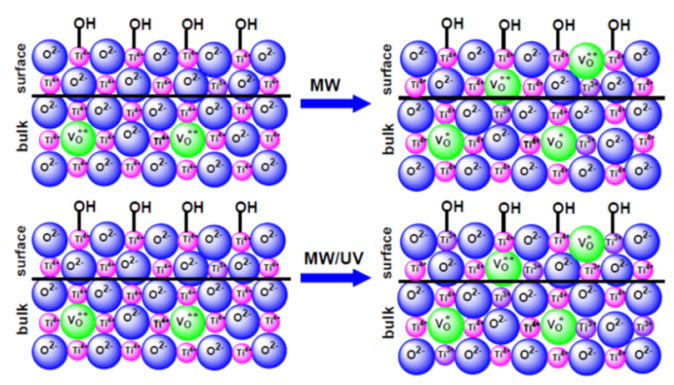

Scheme 1. Simplified view of the effects of microwave and microwave/ultraviolet irradiation on the anatase and rutile polymorphs that lead to formation of $\mathrm{F}$ and $\mathrm{Ti3}+$ color centers. Reproduced from [22]. Copyright 2003 by Elsevier B.V.

Unlike the anatase and rutile polymorphs, only negligible changes occurred in the Raman spectral intensities of $\mathrm{P}-25 \mathrm{TiO}_{2}$ under both $\mathrm{MW}$ and $\mathrm{MW} / \mathrm{UV}$ irradiations. That is, the Raman spectral intensities are severely curtailed by the microwave effect with or without concomitant UV radiation. This is likely the result of the nature of the $\mathrm{P}-25 \mathrm{TiO}_{2}$ heteroarchitecture of a rutile domain (or particle) strongly coupled (fused) to anatase [24] that resulted from the manner by which $\mathrm{P}-25$ is produced in the high temperature $\mathrm{H}_{2}$ flame in the presence of oxygen (precursor, $\mathrm{TiCl}_{4}$ ). The interfacial construct between the rutile and the anatase is perhaps the major defect in $\mathrm{P}-25 \mathrm{TiO}_{2}$ which, under applied microwave radiation fields, likely causes the accumulation of photogenerated charge carriers at this interface (interfacial or space-charge polarization [25]).

In summary, we deduce from the presently available data and the above discussion that the microwave energy absorbed by the $\mathrm{P}-25 \mathrm{TiO}_{2}$ system was consumed to bring about changes at the interfacial boundaries between the coupled rutile and anatase polymorphic structures. Further, such changes may lead to generation of oxygen vacancies, well known to trap conduction band electrons to yield $F$-type color centers, thereby allowing the valence band holes to produce additional ${ }^{\circ} \mathrm{OH}$ radicals as evidenced by the ESR results of Table 1, unlike the anatase and rutile samples where the number of ${ }^{\circ} \mathrm{OH}$ radicals decreased on UV/MW combined irradiation relative to UV irradiation alone. 


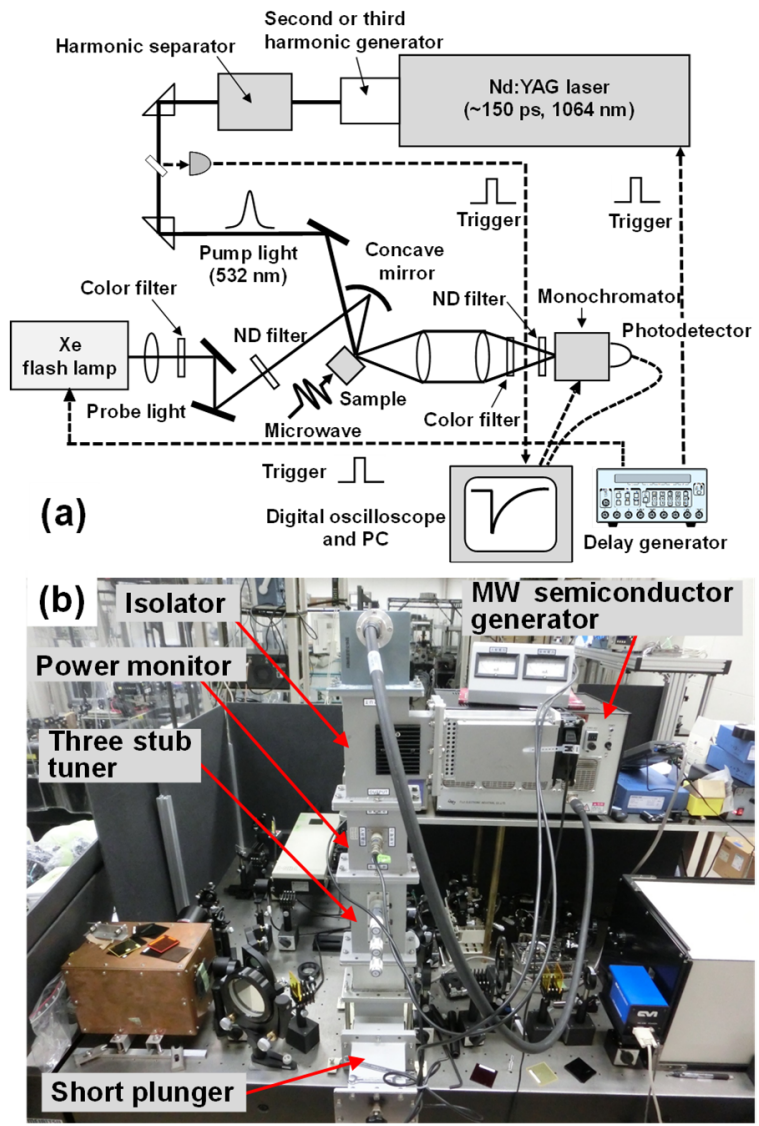

Figure 4. Schematic diagram illustrating the overall diffuse reflectance setup; (b) photograph of the microwave irradiation setup. Reproduced from [26]. Copyright 2015 by Royal Society of Chemistry.

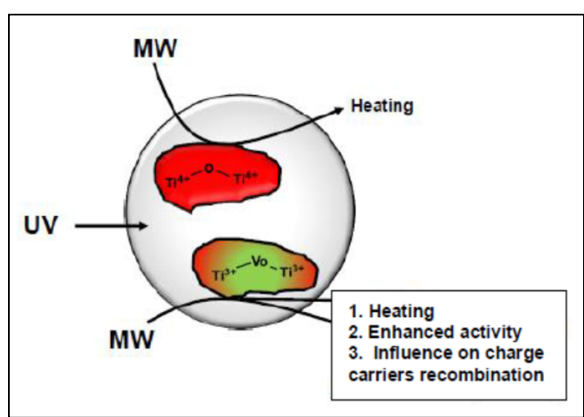

Figure 5. Cartoon depicting the influence of microwave radiation on a regular ST01 particle and on Vo-ST01 particle. Reproduced from [25]. Copyright 2015 by Royal Society of Chemistry. 
The next question is, where does the microwave affect Evonik $\mathrm{P} 25 \mathrm{TiO}_{2}$ particles? Transient decay kinetics at $550 \mathrm{~nm}$ for all three samples were determined in situ using a 150 ps Nd-YAG pulsed laser system $(10 \mathrm{~Hz})$ and a Xe flash lamp (2 ms pulses) probe while samples were being microwave-irradiated (2.45 GHz) [26] (Figure 4). The transient(s) absorbing at the probe wavelength displayed double exponential decay kinetics: a fast decay that occurred within ca. 5-12 ns ascribed to recombination of photogenerated shallowtrapped or free conduction band electrons with valence band holes, and a slower decay that occurred from hundreds of nanoseconds to several microseconds attributable to recombination of electrons trapped in deep traps (e.g., either as $\mathrm{Ti}^{3+}$ or as $\mathrm{F}$ color centers) with free holes.

Contrary to conventional heating, which heats up the titania particle from the surface to the bulk, microwave dielectric heating occurs inside-out from the bulk to the surface while the samples are being simultaneously UV-irradiated. Accordingly, it is not unreasonable to infer that the surface of the titania particles was modified, particularly by the former treatment $(\mathrm{UV} / \mathrm{CH})$. Hence, to the extent that diffuse reflectance spectra probe the surface of the particles, the greater absorption intensity of oxygen vacancies $\mathrm{TiO}_{2}$ subjected to UV/CH is sensible. The presence of oxygen vacancies within the crystal lattice of ceramics has been reported to enhance microwave heating through Joule heating that brings about changes of the microscopic electrical resistance in ceramics. Although conversion of microwave energy to thermal energy may be affected by the presence of oxygen vacancies, it may be possible (under some conditions) to control the recombination of charge carriers and increase the catalyst photoactivity as inferred in Figure 5.

\subsection{Electromagnetic wave effects on living organisms and biomaterials}

The interaction of electromagnetic fields with various life processes has intrigued scientists since the $1800 \mathrm{~s}$. Of current interest are the electromagnetic fields present in microwave radiation, which spans a frequency from $300 \mathrm{GHz}$ to $300 \mathrm{MHz}$ (i.e., from a wavelength of $1 \mathrm{~mm}$ to $1 \mathrm{~m}$ ). Microwaves are used widely in communications and in heating, particularly in the heating of foodstuffs. This non-ionizing electromagnetic radiation is absorbed at the molecular level causing changes in the vibrational energy of the molecules; it also manifests itself as heat [28]. Identifying and evaluating biological effects of microwaves have been rather complex and controversial. In this regard, enzymatic reactions involving microwaves have been the object of active investigations in the latter half of the 20 th century, with the first such study reported in the early 1970s. For instance, microwave radiation to inactivate the enzymes in the brain of animals has been used widely since Stavinoha and coworkers first introduced it in 1970 [29]. Inactivating the enzymes makes it possible to sample and measure many enzymatically destroyed brain neurochemicals thereby reducing the influence of postmortem changes [30]. Usage of microwaves has succeeded in stopping enzymatic activity selectively with minimal damage to other cells and/or proteins under in vivo conditions, for which equipment is commercially available.

Aging and quality improvement of meat [31]: Our research strategy is an attempt to discover phenomena that other energies cannot imitate and apply them to the food sector. In particular, because of the need for control of these precise microwaves, semiconductorbased generators are very effective microwave sources. One experimental example of our research using direct heating of a substance by microwaves and an electromagnetic wave effect is shown in Figure 6. The papain enzyme, used this research as a model enzyme, is a cysteine protease enzyme largely used as a meat tenderizer via the breakdown of tough meat fibers, thereby rendering the meat easier to cook. This enzyme is used extensively in the 
textile, pharmaceutical, and cosmetic industries. If the enzymatic activity of papain could be enhanced by the microwaves' electromagnetic fields, it would lead to significant industrial benefits.

Possible changes in the degree of enzymatic activity in the papain-assisted hydrolysis of proteins present in beef samples were examined by comparing microwave heating relative to conventional heating [4]. The hydrolysis in beef samples whose surface was covered with the papain enzyme was used as the model process $(20 \times 20 \times 50 \mathrm{~mm}$ rectangularly-shaped specimens). The papain-treated beef samples were subjected to pulsed microwave irradiation (PMI; 20 -millisecond pulses microwave irradiation; Figure 6b) and to continuous-wave microwave irradiation (CMI; continuous microwave irradiation Figure $6 \mathrm{c}$ ). Under microwave heating, the papain-treated samples of Figure $6 \mathrm{~b}$ and Figure $6 \mathrm{c}$ softened in a manner suggestive of liquefied jelly when compared to the non-irradiated papain-free initial sample (Figure 6a). That is, it shows that exposing the papain-coated beef specimens to microwave heating enhanced the decomposition of the protein(s) on the beef surface. We also observed that the degree of softness of the beef surface under PMI conditions was, to some extent, greater than under CMI. By contrast, upon being subjected to heating in an electric furnace at $45^{\circ} \mathrm{C}$ for $10 \mathrm{~min}$, the papain-treated beef sample of Figure $6 \mathrm{~d}$ softened much less than under microwave irradiation. Moreover, after the conventional heat treatment, the specimen's surface was unlike the jelly-like surface displayed by the samples of Figure $6 \mathrm{~b}$ and Figure $6 \mathrm{c}$. Experiments were also conducted at $50^{\circ} \mathrm{C}\left(+5^{\circ} \mathrm{C}\right)$ and $40^{\circ} \mathrm{C}\left(-5^{\circ} \mathrm{C}\right)$ in the electric furnace to examine whether errors in temperature measurements could explain this difference. There were no differences at both these temperatures. Accordingly, despite the same temperature conditions $\left(45^{\circ} \mathrm{C}\right)$, the microwaves promoted the activity of papain on the beef surface that we attribute to the microwaves' electromagnetic fields.

Plant growth promotion technology: Microwave-induced growth acceleration of plants is reported in this research. The response of plants (Arabidopsis thaliana) to microwave irradiation was investigated to specify the conditions that enhanced plant growth. Microwave irradiation accelerated the phase transition from vegetative to reproductive growth, the growth of the inflorescence stem, as well as seed germination. However, microwave irradiation did not affect plant diameter. In addition, it was found that microwave irradiation enhanced the expression of a gene that regulates growth phase transition, suggesting that microwave irradiation could indeed enhance the growth of plants by modulating the expression of gene. On the other hand, the expression of genes involved in the response of plants to heat stress were not clearly altered by microwave irradiation. Our results suggest that the effects of microwave irradiation on plant growth is not from the thermal effect. By understanding the key factor behind the microwave-dependent growth stimulation, it makes it possible to apply this finding to the growth of other plants (potato, etc). 


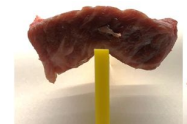

(a)

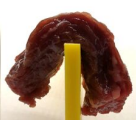

(b)

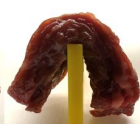

(c)

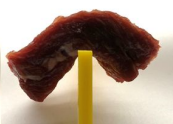

(d)

Figure 6. Observations of the surface changes and the degree of beef samples after applying papain to the beef: (a) control experiment with no papain used on the beef, (b) papain-treated beef sample after heating with pulsed microwave irradiation (PMI) and maintaining it at $45^{\circ} \mathrm{C}$ for $10 \mathrm{~min}$ (c) papain-treated beef sample subjected to continuous microwave irradiation (CMI) under otherwise identical conditions as in (b), and (d) papain-treated beef sample subjected to heating in an electric furnace at $45^{\circ} \mathrm{C}$ for 10 min. Reproduced from [33]. Copyright 2019 by Nature Springer.

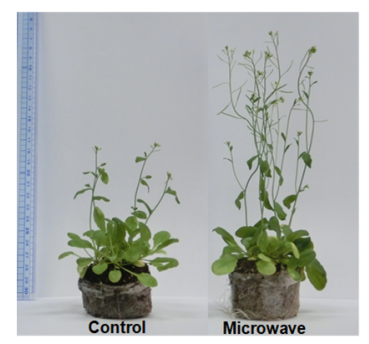

Figure 7. Grown comparison of Arabidopsis thaliana with no radiation (control) and microwave radiation (Microwave)

When either microwave heating or conventional heat treatment was applied to A thaliana, a significant change in the growth of the leaves could not be observed. However, the shift to the reproductive stage and the growth of the inflorescence stem were dramatically promoted in the microwave treatment condition (Figure 7). Because such phenomena were not observed under ordinary high temperature $\left(40^{\circ} \mathrm{C}\right)$ conditions, a specific effect that was only observed in the case of microwave irradiation was expected. When the expression of the gene that regulates growth or stress response was investigated, the expression of the gene which controls the growing stage was affected by microwave irradiation.

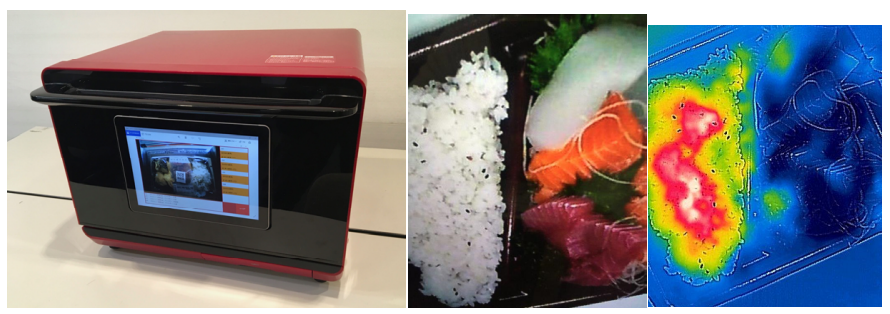

Figure 8. (Top) Photo of intelligent cooker, (bottom left) photo of Sushi (Raw fish) lunch box, (bottom right) The rice warms up to the set temperature and the sushi (raw fish) doesn't warm up at all (thermographic image). 
Intelligent cooker: Currently, eating habits taking advantage of individual personality advances, food of various kinds and form is being sold. On the other hand, the advances of food heating method is not added to most meals. Especially the microwave oven is convenient to heat cooked food in supermarkets and convenience stores. However, it is impossible for individuals to set the temperature, and heating of the microwave oven is adjusted by the arrangement on the food, water concentration and salt concentration. In order to practice this innovation, we switched the microwave source of the microwave oven to the RF high power semiconductor generator (semiconductor type generator) (Figure 8). There have been attempts to utilize semiconductor type generator for microwave from long ago, but there was no one available with the size and price of the device, and it could not be put into practical use. However, in recent years we succeeded in ultra-miniaturization of the generator, and it is estimated that selling price will be sold at the same price as the current price if mass-produced. We will introduce "intelligent cooker" such as new microwave cooking oven and new warm catering cart that operate by partial heating of foods in lunch box and hospital plate meal using semiconductor type generator. Furthermore, it also shows that combinations with internet of things (IoT) are effective when implementing and sale at a market.

\section{References}

[1] C. F. Buchholz, Catechismus d. Apothekerkunst. Erf. 1810, Vermehrt herausgeg. v. Brandes. Erfurt 1820, 2 Bde.

[2] J. G. Calvert, J. N. Pitts, Photochemistry. Wiley \& Sons; New York, USA, 1966.

[3] S. Horikoshi, R. F. Schiffmann, J. Fukushima, N. Serpone, Microwave chemical and materials processing, A tutorial. Springer, Japan, 2017; ISBN 978-981-10-6465-4.

[4] M. A. Rodriguez, P. Prieto, A. de la Hoz, Ý. Diaz-Ortiz, D. R. Martin, J. I. Garcia, ChemistryOpen, 2015, 4, 308-317.

[5] J. Ma, J. Phys. Chem. A, 2016, 120, 7989-7997.

[6] T. Razzaq, J. M. Kremsner, C. O. Kappe, J. Org. Chem., 2008, 73, 6321-6329.

[7] S. Horikoshi, N. Serpone, Catal. Today, 224, 225-235 (2014).

[8] S. Horikoshi, H. Tsutsumi, H. Matsuzaki, A. Furube, A. V. Emelinec, N. Serpone, J. Mater. Chem. C, 2015, 3, 5958-5969.

[9] F. Kishimoto, T. Imai, S. Fujii, D. Mochizuki, M. M. Maitani, E. Suzuki, Y. Wada, Sci. Rep., 2015 5, 11308.

[10] S. Horikoshi, T. Watanabe, A. Narita, Y. Suzuki, N. Serpone, Sci. Rep., 2018, 8:5151, DOI:10.1038/s41598-018-23465-5.

[11] K. Hashimoto, H. Irie, A. Fujishima, Jpn. J. Appl. Phys., 2005, 44, 8269-8285.

[12] K. Kabra, R. Chaudhary, R. L. Sawhney, Ind. Eng. Chem. Res., 2004, 43, 7683-7696.

[13] K. Konstantinou, T. A. Albanis, Appl. Catal. B: Environ., 2003, 42, 319-335.

[14] D. S. Bhatkhande, V. G. Pangarkar, A. A. Beenackers, J. Chem. Technol. Biotechnol., 2002, 77, 102-116. 
[15] A. Fujishima, K. Hashimoto, T. Watanabe, $\mathrm{TiO}_{2}$ Photocatalysis: Fundamentals and Applications; BKC Incorporated: Tokyo, Japan, 1999.

[16] S. Horikoshi, H. Hidaka, N. Serpone, Environ. Sci. Technol., 2002, 36, 1357-1366.

[17] S. Horikoshi, H. Hidaka, N. Serpone, Environ. Sci. Technol., 2002, 37, 5813-5822.

[18] S. Horikoshi N. Serpone, J. Photochem. Photobiol. C: Photochem. Rev., 2009, 10, 96110.

[19] S. Horikoshi, F. Sakai, M. Kajitani, M. Abe, N. Serpone, Chem. Phys. Lett., 2009, 470, 304-307.

[20] S. Horikoshi, A. Matsubara, S. Takayama, M. Sato, F. Sakai, M. Kajitani, M. Abe, N. Serpone, Appl. Catal. B: Environ., 2009, 91, 362-367.

[21] S. Horikoshi, H. Hidaka, N. Serpone, Chem. Phys. Lett., 2003, 376, 475-480.

[22] S. Horikoshi, M. Abe, S. Sato, N. Serpone, J. Photochem. Photobiol. C: Photochem. Rev., 2011, 220, 94-101.

[23] V. N. Kuznetsov, N. Serpone, J. Phys. Chem. C, 2009, 113, 15110-15123.

[24] S.A. Chambers, C. M. Wang, S. Thevuthasan, T. Droubay, D. E. McCready, A. S. Lea, V. Shutthanandan, C. F. Windisch, Thin Solid Films, 2002, 418, 197-210.

[25] S. K. Arora, V. Patel, B. Amin, A. Kothari, Bull. Mater. Sci., 2004, 27, 141-147.

[26] S. Horikoshi, H. Tsutsumi, H. Matsuzaki, A. Furube, A. V. Emeline, N. Serpone, J. Mater. Chem. C, 2015, 3, 5958-5969.

[27] A. V. Emeline, L. G. Smirnova, G. N. Kuzmin, L. L. Basov, N. Serpone, J. Photochem. Photobiol., A, 2002, 148, 97-102.

[28] S. Banik, S. Bandyopadhyay, S. Ganguly, Bioresource Technol., 2003, 87, 155-159.

[29] W. B. Stavinoha, B. Pepelko, P. W. Smith, Pharmacologist ,1970, 12, 257.

[30] W. B. Stavinoha, S. T. Weintraub, A. T. Modak, J. Neurochem., 1973, 20, 361-371.

[31] S. Horikoshi, K. Nakamura, M. Yashiro, K. Kadomatsu, N. Serpone, Sci. Rep., 2019, 9:8945 | https://doi.org/10.1038/s41598-019-45152-9. 